\title{
Real-Time Maintenance Optimization Considering Health Monitoring and Additive Manufacturing
}

\author{
Adrian Cubillo ${ }^{1}$, Suresh Perinpanayagam ${ }^{2}$, Manuel Esperon-Miguez ${ }^{3}$ and Philip John ${ }^{4}$ \\ ${ }^{1,2,3,4}$ Cranfield University, College Road, Cranfield MK43 OAL, United Kingdom \\ a.cubillo@cranfield.ac.uk \\ suresh.nayagam@cranfield.ac.uk \\ m.esperonmiguez@cranfield.ac.uk \\ p.john@cranfield.ac.uk
}

\begin{abstract}
Prognostics Health Management (PHM) and Integrated Vehicle Health Management (IVHM) are extensive areas of research. Whereas a lot of work has been done in diagnostics and prognostics, economic viability is an important consideration. The availability of aircraft in the aerospace sector is a critical factor. Thus, cost and downtime are the main parameters to assess the impact of IVHM. Additionally, new repair technologies, such as additive manufacturing (AM), have the potential to become standard repair procedures, complementing IVHM, and its viability also has to be assessed. However, to accurately study the impact of these factors, the characteristics of aerospace maintenance have to be taken into account. Several approaches are followed in aircraft maintenance, depending on cost, downtime and aircraft availability constraints. For instance, some parts can be repaired on the ground and assembled again on the same aircraft, while single Line Replaceable Units (LRUs) need to be removed, replaced and later repaired in the workshop without affecting the availability of the aircraft. With the gradual introduction of IVHM, the viability of any new IVHM technology needs to be assessed.

This paper describes an extensive cost and downtime model to take into account all these scenarios, including the impact of using different types of IVHM systems. The impact of IVHM and new repair technologies is discussed comparing maintenance cost and downtime of parts of LRUs and parts repaired when the aircraft is on the ground.

Secondly, a real-time maintenance case study based on IVHM, a cost and downtime model and additive manufacturing is presented. This application allows the optimization of maintenance activities by updating the available resources and their corresponding cost and time, along with the actual prediction of the Remaining Useful
\end{abstract}

Cubillo et al. This is an open-access article distributed under the terms of the Creative Commons Attribution 3.0 United States License, which permits unrestricted use, distribution, and reproduction in any medium, provided the original author and source are credited.
Life (RUL) using a health monitoring system, instead of depending on historical component/sub-system failure probabilities.

\section{INTRODUCTION}

Integrated Vehicle Health Management (IVHM) refers to several aspects of the vehicle and its management and aims to assess the current and future health state integrated within a framework of available resources and operational demands. Along with other research areas, it covers the consideration of available resources and operational demands of the whole fleet (Jennions, 2011). The concept of IVHM can be divided into several sub-areas. The architecture of IVHM systems follows the Open System Architecture for Condition-Based Maintenance (OSA CBM) (Dunson \& Harrington, 2008; Xia et al., 2010). Following this classification, the approach presented in this paper is at level of "advisory generation".

The benefit of IVHM for the maintainer is normally shown in the reduction of maintenance cost and increased availability of the fleet. This is due to reduced maintenance time thanks to diagnostics capabilities that allow fault localization and fault isolation early on in the operation, the reduced cost and time of planned maintenance operations and the avoided cost of secondary damage (Esperon-Miguez, 2013).

However, these benefits should be assessed against the potential drawbacks of having an IVHM system, e.g. extra costs include the remaining life of the component when it is replaced before it fails and the cost of implementing the IVHM system. Additionally, false alarms can lead to unnecessary inspections with the subsequent extra cost and downtime. Undetected failures would also lead to additional costs and downtime. The effect of these factors in the availability of the fleet was analyzed by Datta and Squires (2004).

Aircraft maintenance can be divided into maintenance planning and execution of maintenance. The former, 
commonly referred to as Continuing Airworthiness Management Organization (CAMO, EASA, 2003), can be the airline or the Maintenance Repair and Overhaul (MRO) provider, while the latter is commonly the MRO provider. The approach presented in this paper falls into maintenance planning or CAMO.

A maintenance operation is triggered by the CAMO when a fault is detected during routine inspection or using an IVHM system. In terms of maintenance, there is an important difference between Line Replaceable Units (LRUs) and independent components. LRUs are not repaired while the aircraft is on the ground; instead, damaged LRUs are replaced to minimize downtime. These are then inspected and repaired without affecting the availability of the aircraft in the workshop and installed in another aircraft (Kumar \& Varkey, 2012). However, for simpler faulty systems of independent components, the inspection and repair processes can be done while the aircraft is on the ground.

This paper presents an approach to estimate the cost and downtime of an aircraft fleet based on the methodology developed by Esperon-Miguez (2013) with additional functionalities to consider not only the effect of IVHM, but also the consideration of using Additive Manufacturing (AM) as a repair procedure instead of traditional methods. AM is a new technology with potential to be used in the aerospace sector (Uriondo et al., 2014). IVHM and AM will have a different impact in terms of cost and downtime, depending on the type of maintenance, i.e. whether the damaged component is part of an LRU or not.

A comprehensive description of the maintenance cost and downtime model is presented in Section 2. The maintenance optimization case study considering health monitoring and AM systems are described in Section 3. This real-time health monitoring model will calculates the cost and downtime of the different repair procedures and IVHM systems. The benefits of IVHM and flexible repair processes are discussed in Section 3. The results of the case studies are introduced in Section 4 and the discussion and conclusions of the results and the real-time approach are presented in Section 5 .

\section{COST AND DOWNTIME MODEL}

\subsection{Model Overview}

This model focuses on a specific component/failure mode and calculates the cost of its maintenance based on: probability of failure, whether there is a health condition monitoring tool or not, the repair procedure and its associated costs and times, which can be the traditional repair method or a novel procedure, e.g. AM. The algorithm does not aim to evaluate the maintenance cost of the whole aircraft or LRU.

First, the scenarios are defined (Sub-section 2.2). Then, the probabilities are described (Sub-section 2.3), followed by the maintenance costs (Sub-section 2.4) and times (Sub- section 2.5). Finally, the computation of the total cost and downtime is described in Sub-section 2.6.

\subsection{Scenarios.}

This section introduces the different scenarios that will be later compared and discussed. Three possible IVHM scenarios are considered:

- Scenario IVHM-1: No health condition monitoring tool is installed in the component.

- Scenario IVHM-2: A health condition monitoring tool capable of detecting failures prior to total failure (shortterm prognosis) but not preventing failure during operation.

- Scenario IVHM-3: A health monitoring tool capable of long term prognosis; thus, allowing for scheduled maintenance.

Please note short-term prognosis and long-term prognosis terminology is used in this paper instead of diagnostics and prognostics. This is because diagnostics encompasses both fault detection and isolation. In this case study we assume that we have already identified the precursor which is related to the fault. The only variable considered in the case study is whether the failure will occur in a short duration or a long duration for scheduling the maintenance.

Regarding the repair procedure of the damaged component, 4 scenarios are considered:

- Scenario Repair-1: The whole LRU is replaced by a new one and the faulty LRU is repaired and ready to be re-installed in a new aircraft in less than 30 days. The repair procedure consists of traditional methods (no AM). Therefore, the repair and inspection of the LRU does not affect the downtime of the aircraft.

- Scenario Repair-2: Identical to Scenario Repair-1 but the part is repaired using AM.

- Scenario Repair-3: The part is repaired and reinstalled while the aircraft is on the ground. Traditional repair procedures are considered and the part is replaced in the same aircraft. Mean Time To Repair (MTTR) is a critical factor as the availability of the aircraft is compromised.

- Scenario Repair-4: The part is repaired and reinstalled while the aircraft is on the ground as in Repair-3 by using AM. MTTR is a critical factor because it affects the availability of the aircraft.

All the possible combinations of IVHM and repair scenarios, i.e. a total of 12 scenarios, are analyzed and discussed in the following sections. 


\subsection{Probabilities}

This section defines all the probabilities that have to be defined for the model regarding the failure (input probabilities) and the probabilities that define the characteristics of the IVHM system (IVHM probabilities).

\section{Input Probabilities}

The model is based on a set of probabilities. In this section, all the probabilities considered by the model are presented. It should be noted that the case studies will be based on commercial aircraft and some of these probabilities are only relevant for military vehicles. The input probabilities, summarized in Table 1, are as follows:

Table 1. Input Probabilities

\begin{tabular}{l|l}
\hline Abbreviation & Description \\
\hline P_S & $\begin{array}{l}\text { Probability of component failure per flying } \\
\text { hour }\end{array}$ \\
\hline P_VL & Probability of catastrophic failure \\
\hline P_MF & $\begin{array}{l}\text { Probability of being unable to complete the } \\
\text { mission }\end{array}$ \\
\hline P_MA & $\begin{array}{l}\text { Probability of aborting the mission due to a } \\
\text { failure alarm }\end{array}$ \\
\hline P_RC & $\begin{array}{l}\text { Probability of the failure causing reduced } \\
\text { capability for future missions }\end{array}$ \\
\hline P_RA & $\begin{array}{l}\text { Probability of the failure causing loss of } \\
\text { availability }\end{array}$ \\
\hline P_RDA & $\begin{array}{l}\text { Probability of the } \\
\text { diagnosis/repair/replacement resulting in a } \\
\text { loss of availability }\end{array}$ \\
\hline P_CA & $\begin{array}{l}\text { Probability of loss of availability due to the } \\
\text { check of the system due to an alarm }\end{array}$ \\
\hline P_no_stock & $\begin{array}{l}\text { Probability of not having stock of the faulty } \\
\text { component on the ground }\end{array}$ \\
\hline
\end{tabular}

- Probability of failure (P_S): Defines the chances of having a component failure. Because it is defined as probability per flying hour, all the total costs and downtime generated by the algorithm will also be obtained per flying hour. It is considered to be $8.10^{-6}$.

- Probability of losing the vehicle due to the failure $\left(\mathbf{P}_{-} \mathbf{V L}\right)$ : This probability is only taken into account if a failure occurs and it is not predicted by the IVHM system. The case is considered catastrophic and values in terms of cost and downtime are irrelevant. It is considered 0 for the case studies because the component examined is not safety critical.

- Probability of not completing the mission due to the failure (P_MF): The probability of not completing the mission due to the failure is considered if the failure occurs, is undetected by the prognostics IVHM system and no catastrophic failure occurs but the mission cannot be completed. The case studies assume that the plane is capable of finishing the mission. Thus, the probability $\mathrm{P}_{-} \mathrm{MF}$ is set to 0 .
- Probability of aborting the mission due to a failure alarm (P_MA): This is the probability of not completing a mission due to a failure alarm, even if it is a false alarm. This probability is computed only if there is no failure and a false alarm occurs. For the same reason as $\mathrm{P}_{-} \mathrm{MF}$, this value is set to 0 .

- Probability of reduced capability for future missions (P_RC): This is the probability of the failure affecting future missions, e.g. if the part has to be repaired or the component replaced in order to complete the next mission. It is assumed to occur always in the presented case studies. It is set to 1 .

- Probability of the repair/replacement resulting in a loss of availability (P_RA): This is the probability of having the plane on the ground or the aircraft not being available (loss of availability), e.g. a cancelled flight because the component is being replaced or repaired. This value is set to 0.35 for all scenarios.

- Probability of the diagnosis/repair/replacement resulting in a loss of availability (P_RDA): This probability is identical to the previous $P_{-}$RA but due to an undiagnosed failure. It is also set to $0 . \overline{3} 5$.

- Probability of the check of the component resulting in a loss of availability (P_CA): This probability considers the chances of a check of the system resulting in a loss of availability. It applies when no failure occurs but a false alarm by the IVHM system leads to the inspection of the component. For the current case study, the P_CA has been set to 0.05 for all the scenarios. It should be kept in mind that this loss of availability primarily depends on the ratio of false alarms of the IVHM system.

- Probability of not having stock (P_no_stock): This new probability has been incorporated to take into account additional costs if no stock is available on the ground when a part has failed and needs to be replaced on the ground. This applies to replacements of the faulty component (Repair-3 and 4) and replacement of the whole LRU (Repair-1 and 2).

For the current case study, it has been considered that the probability of having stock of a LRU is higher than for a spare sub-component. Therefore, the probability for Scenarios Repair-1 and 2 (LRU) is 0.05 and 0.1 for Repair-3 and 4 (component replaced on the ground).

\section{IVHM Probabilities}

An IVHM system can be assessed from a cost analysis perspective by defining the following parameters (EsperonMiguez, 2013), see Table 2 
Table 2. IVHM Probabilities

\begin{tabular}{l|l}
\hline Abbreviation & Description \\
\hline P_LP & $\begin{array}{l}\text { Probability of failure occurring and being } \\
\text { undetected by long-term prognostics }\end{array}$ \\
\hline P_SP & $\begin{array}{l}\text { Probability of failure occurring and being } \\
\text { undetected by short-term prognostics }\end{array}$ \\
\hline P_FN & Probability of false negative \\
\hline P_FA & Probability of false alarm \\
\hline
\end{tabular}

- Probability of failure occurring and being undetected by long-term prognostics $\left(\mathbf{P}_{-} L P\right)$ defines the chances of not predicting a failure early enough to plan the maintenance in advance.

This parameter is set to 1 for IVHM-1 and 2: scenarios in which no long-term prognostics capability is installed, For IVHM-3, which refers to the system with prognostics capabilities, this value is set to 0.01 .

- Probability of failure occurring and being undetected by short-term prognostics (P_SP) defines the chances of not detecting the failure by short-term prognostics.

It should be noted that detecting a failure just before it occurs is expected to be more probable than long in advance ( $\mathrm{P}_{-}$LP). Therefore, this value is 1 for IVHM-1 and $10^{-4}$ for IVHM-2 and 3 .

- Probability of false negative (P_FN) defines the rate of failures that are undetected by the IVHM system.

The detection of a failure after it has occurred is also expected to be more probable than detection in advance. Thus, it is set to $10^{-5}$ for IVHM- 2 and 3 and at 1 for IVHM-1.

- Probability of false alarm (P_FA) defines the probability of an alarm by the IVHM system that will trigger maintenance actions when there is no actual fault in the system.

This probability is particularly critical and should be kept to a minimum. It should be noted that it is computed against ( $1-P_{-} S$ ), not against $P_{-} S$ as the previous ones. Thus, it is much lower: $10^{-15}$ for IVHM2 and 3 and 0 for IVHM-1 because no IVHM system is installed.

\section{Case Probabilities}

This sub-section describes the probability of each possible "case", which is a function of the "input probabilities" defined in the previous sub-section. The cases are defined sequentially depending on:

- Whether there is a failure or not;

- The reaction by the IVHM system: prognosis, diagnosis, undetected, false negative, false alarm; and
- The effect of the failure: vehicle loss, mission loss, future missions affected, availability affected.

All the possible outcomes are shown in Figure 1, where the combination of probabilities for each case is shown.
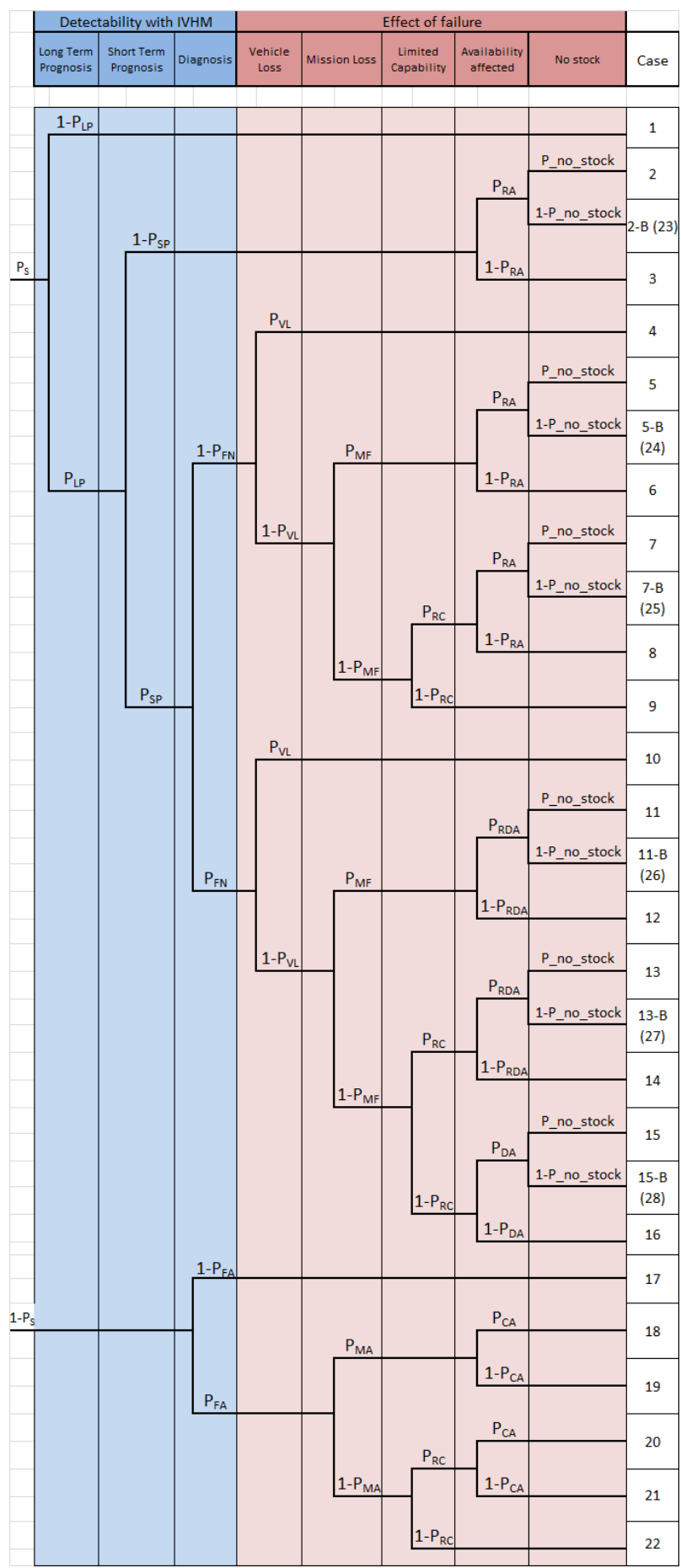

Figure 1. Case probabilities as a function of input probabilities following Eq. (1)

The probability of each case is obtained using Eq. (1). 


$$
P_{i}=\prod P_{j} \text { being }\left\{\begin{array}{c}
j=\text { Prob.of step in the path } \\
i=\{1-28 \text { cases }\}
\end{array}\right.
$$

\subsection{Input Costs}

This section describes the costs associated with maintenance, the use of AM, loss of availability, compensation, etc. The different types of costs that the user can modify for each specific failure mode/component are described as follows (see Table 3):

Table 3. Input Costs

\begin{tabular}{l|l}
\hline Abbreviation & Description \\
\hline C_SC & Scheduled maintenance component cost \\
\hline C_UC & Unscheduled maintenance component cost \\
\hline C_SCL & Scheduled labour cost per hour \\
\hline C_UCL & Unscheduled labour cost per hour \\
\hline C_RULL & $\begin{array}{l}\text { Cost of prognostics remaining life lost } \\
\text { (long-term prognostics) }\end{array}$ \\
\hline C_RULS & $\begin{array}{l}\text { Cost of prognostics remaining life lost } \\
\text { (short-term prognostics) }\end{array}$ \\
\hline C_FA & Extra cost due to false alarms \\
\hline C_C & Compensation costs \\
\hline C_SD & Secondary damage cost \\
\hline C_FT & Replaced part flight test cost \\
\hline C_LI & Loss of income \\
\hline C_LO_LRU & $\begin{array}{l}\text { Cost of logistics to send LRU from aircraft } \\
\text { to workshop (LRU only) }\end{array}$ \\
\hline C_R_LRU & $\begin{array}{l}\text { Cost of repairing damaged part in the } \\
\text { workshop (LRU only) }\end{array}$ \\
\hline C_LA_LRU & Cost of labour in workshop (LRU only) \\
\hline
\end{tabular}

- Scheduled Maintenance Component cost (C_SC): Represents the cost of the new spare/repaired part when the replacement has been planned (scheduled). If the part is a sub-component of a LRU, this cost is not associated with the whole LRU and has to be set to 0 . The cost of the repairing it in the workshop will be described later (see C_R_LRU).

This assumption implies that the value of the new LRU will be identical to the value of the faulty LRU once the damaged component is repaired or replaced at the workshop. The costs for the case studies are set to 20 MUs (Monetary Units) for Repair-3, 4 and 0 for Repair$1,2$.

- Unscheduled Maintenance Component cost (C_UC): Is identical to the previous cost, in the sense that it refers to the cost associated with the replacement of the component. However, it refers to the cost of the part when it is an unscheduled replacement. The reason is that the cost could be higher if the replacement is not expected; e.g. tight delivery times lead to higher costs.

As with the previous parameter, for LRU units, C_UC is set to 0 because the cost of repairing the component in the workshop is defined by C_R_LRU (Repair-1, 2). For Repair-3, 4, C_SC is set to $30 \mathrm{MUs}$.

- Scheduled labour cost (C_SCL): The replacement of a part or LRU on the ground will lead to a labor costs that are affected by this parameter along with the average repair time. This cost can vary between Repair3, 4 and Repair-1, 2 because the LRU may require more resources, e.g. 3 technicians instead of 2 . Additionally, if AM is used, the labour cost may change as well.

For the case studies, the replacement of the LRU unit is not affected by using AM. Thus, C_SLC for Repair-1 and 2 are identical and set to 3 MUs per hour. While for Repair-3, 4 it is considered that less labour is needed if AM is used, 2 MUs per hour (Repair-3), and 3 MUs per hour for Repair-4.

- Unscheduled labour cost (C_UCL): Identical to the previous parameter C_SLC but with a higher cost due to the fact that it is unplanned maintenance. It is $5 \mathrm{MUs}$ per hour for Repair-1, 2, 3 and 4 MUs per hour for Repair-4.

- Cost of remaining life lost using long-term prognostics (C_RULL): It is necessary to take into account the value of the part that is lost due to replacing it before it actually fails. This means that there is a percentage of healthy life that is not used when longterm prognostics is used.

This cost is identical for all the scenarios and set to 5 MUs (even when no IVHM system is installed). It should be noted that the difference between different IVHM systems will not be reflected on this cost but on the probability of detecting the failure in advance (P_LP).

- Part false alarm cost (C_FA): Represents the cost of the part when a false alarm occurs and it is necessary to take into account the costs caused by an ineffective IVHM system, e.g. an IVHM system that triggers alarms when no failure has occurred. This cost takes into account the cost of replacing the part when a false alarm has occurred.

This cost implies the assumption of no second inspection on the ground, meaning that the technicians will replace the component without further inspection. For a LRU, the part is repaired in the workshop and the non-existent fault will be detected and no additional repair operations will be done. Therefore, C_FA should be 0 for Repair 1, 2 and 20 MUs for Repair 3, 4 .

- Compensation cost (C_C): Only applies from an MRO organization perspective, and takes into account the compensation cost in terms of the penalty that the MRO organization has to pay if availability expectations are not met in an availability-based contract. For the case study, this value is set to 6 MUs for all the scenarios. 
- Secondary damage cost (C_SD): It is necessary to consider additional damage to adjacent components due to the initial failure. This damage is assumed to occur if the failure occurs (if the failure is undetected or diagnosed). However, if long/short-term prognostics detect the failure in advance, this cost can be avoided.

It is important to mention that one of the main advantages of short-term prognostics is that even if maintenance cannot be planned, a significant cost reduction due to avoiding this "secondary damage cost" can be obtained. For our case study, this value is set to 30 MUs.

- Flight test cost (C_FT): Takes into account the cost associated with flight tests when the new part is installed in the aircraft. Based on previous work, this value has been set to $2 \mathrm{MUs}$ for all the possible scenarios.

- Loss income (C_LI): Represents compensation to the passengers due to excessive delays (downtime). This cost can be significant. However, the cost analysis of this paper is done from the CAMO's perspective and C_LI is set to 0 because it does not affect the costs of the MRO organization unless it is stated in the MROairline contract.

All the previous costs are associated with actions on the ground while the aircraft is on the ground. However, there are additional costs that only apply to LRUs (Repair-1,2):

- Logistics cost for LRU only (C_LO_LRU): Considers the cost of shipping the faulty LRU to the MRO workshop specialized in that LRU and shipping it back to another aircraft. C_LO_LRU is set to 2 MUs (Repair-1, 2 only).

- Repair cost for LRU only (C_R_LRU): Takes into account the repair of the damaged part inside the LRU once it has been shipped to the workshop. For Repair-1, C_R_LRU is set to 20 MUs and for Repair-2, when AM is used, C_R_LRU is set to 15 . Thus, assuming that less material is required, the repair cost is lower if $\mathrm{AM}$ is used. It should be noted that the assumption of lower cost of the repair process when AM is used is a hypothesis that has not been proven.

- Labour cost for LRU only (C_L_LRU): Takes into account the labour cost of the repair and inspection in the workshop (not on the ground). It includes all the labour costs since the LRU arrives at the workshop until it is shipped to a new aircraft.

For the case study, it is considered identical to the cost of repairing the component on the ground, being lower if AM is used (2 MUs instead of $3 \mathrm{MUs}$ ) for Repair-2 because it is assumed that an AM repair procedure is highly automated and does not require highly-skilled technicians.

\subsection{Timings}

The input times include all the necessary parameters that define the time of each task that may affect the maintenance cost and downtime of the aircraft.

Whether or not these times affect the downtime of the aircraft will depend of the "case" that is considered, e.g. MTTD is considered as downtime if there is an undetected failure but it does not affect downtime if the maintenance task is scheduled. The input times are described as follows (see Table 4):

Table 4. Input Times

\begin{tabular}{l|l}
\hline Abbreviation & Description \\
\hline MTTR & Mean time to repair (the failure) \\
\hline $\begin{array}{l}\text { Check-out time } \\
\text { (T_Check) }\end{array}$ & $\begin{array}{l}\text { Mean time to conduct the necessary } \\
\text { checks }\end{array}$ \\
\hline MTTD & Mean time to detect the failure mode \\
\hline Localization time (T_L) & Mean time to localize the failure \\
\hline Technical delay time & $\begin{array}{l}\text { Mean time delay due to technical } \\
\text { issues }\end{array}$ \\
\hline $\begin{array}{l}\text { Administrative delay time } \\
\text { (T_Adm) }\end{array}$ & $\begin{array}{l}\text { Mean time delay due to } \\
\text { administrative issues }\end{array}$ \\
\hline $\begin{array}{l}\text { Logistics delay time } \\
\text { (T_LO) }\end{array}$ & $\begin{array}{l}\text { Mean time delay due to logistics on } \\
\text { the ground (assuming stock) }\end{array}$ \\
\hline MTTR_LRU & $\begin{array}{l}\text { Mean time to repair (the failure) in } \\
\text { the workshop (LRU only) }\end{array}$ \\
\hline $\begin{array}{l}\text { Localization_LRU } \\
\text { (T_L_LRU) }\end{array}$ & $\begin{array}{l}\text { Mean Time to localize the failure in } \\
\text { the workshop (LRU only) }\end{array}$ \\
\hline MTTD_LRU & $\begin{array}{l}\text { Mean time to detect (the failure) in } \\
\text { the workshop (LRU only) }\end{array}$ \\
\hline $\begin{array}{l}\text { No_stock_delay } \\
\text { (T_no_stock) }\end{array}$ & $\begin{array}{l}\text { Mean time to obtain the part/LRU if } \\
\text { there is no stock on the ground }\end{array}$ \\
\hline
\end{tabular}

- Mean Time To Repair (MTTR): Accounts for the average time required to repair the given failure mode. It only includes the actual time required to repair it and not the time to detect it, localize it or check it.

The scenarios differ between MTTR in Repair-1, 2 and MTTR in Repair-3, 4. The former is set to 2 Time Units (TUs) because the task simply consists of replacing the whole LRU, while the latter is set to $10 \mathrm{MUd}$ because the damaged component has to be replaced or repaired.

- Check-out time (T_check): Considers the time required to check that the maintenance actions have successfully solved the problem and the aircraft is airworthy. This value has been considered constant and set to 3 TUs for all the scenarios

- Mean Time To Detect (MTTD): Refers to the time required to identify the failure mode. It should be noted that for some systems, MTTD and localization time are equivalent and only one of them should be defined.

MTTD is set to 5 TUs for scenarios Repair-3, 4. For Repair-1 and 2, the LRU is not inspected; it is simply replaced. Thus, MTTD is 0 . In reality, this may not be 
zero as various test are done before removing a LRU unit.

- Localization time (T_L): Refers to the time required to localize a failure on the ground, i.e. to find which specific component is affected.

The case study considers that the localization of the failure is relatively simple compared to the detection of the failure mode. Therefore, the localization time is set to 1 TU for Repair-3, 4. For Repair-1 and 2, it is set to 0 because the localization time does not apply to the LRU on the ground.

- Technical, administrative and logistic delay times: These three times all refer to delays in the maintenance operation on the ground due to technical, administrative and logistic delays but they are all treated by the model in the same way.

It is considered that the technical delay is more critical for parts replaced on the ground than if the LRU is replaced because replacing a LRU is a more standard procedure. Additionally, repairing the component on the ground using AM will lead to additional certification procedures and because the process would not be standard, more administrative delays are expected. The logistic delay time is considered to be relatively low because it only refers to logistic delays on the ground and not waiting times for parts that are not in stock (see Table 5 for specific parameter values).

Table 5. Technical, Administrative and Logistic Delays for All the Scenarios

\begin{tabular}{|l|c|c|c|c|}
\cline { 2 - 5 } \multicolumn{1}{c|}{} & Repair-1 & Repair-2 & Repair-3 & Repair-4 \\
\cline { 2 - 5 } \multicolumn{1}{c|}{} & \multicolumn{4}{c|}{ Technical_delay_time (TUs) } \\
\hline IVHM-1 & 1 & 1 & 3 & 3 \\
\hline IVHM-2 & 1 & 1 & 3 & 3 \\
\hline IVHM-3 & 1 & 1 & 3 & 3 \\
\hline IVHM-1 & 1 & 1 & 1 & 1.5 \\
\hline IVHM-2 & 1 & 1 & 1 & 1.5 \\
\hline IVHM-3 & 1 & 1 & 1 & 1.5 \\
\hline & Repair-1 & Repair-2 & Repair-3 & Repair-4 \\
\cline { 2 - 6 } \multicolumn{1}{|c|}{} & 1 & logistic_delay_time (TUs) \\
\hline IVHM-1 & 1 & 1 & 1 & 1 \\
\hline IVHM-2 & 1 & 1 & 1 & 1 \\
\hline IVHM-3 & 1 & 1 & 1 & 1 \\
\hline
\end{tabular}

- Delay time caused by no stock available (no_stock_delay): Accounts for the scenarios in which no stock is available on the ground. This condition is not required in the workshop.

This parameter defines the standard delivery time when there is no stock and its value is set to 24 TUs for all the scenarios.

The following times only apply to actions in the workshop, not on the ground. Therefore, they are only applied to Repair-1, 2. The times are shorter than equivalent operations on the ground because an optimized process is expected in a specialized workshop.

- Mean time to repair in the workshop (LRU only): Accounts for the repair time in the workshop. Therefore, it only applies to RepAIR-1, 2 and it is set to 7 TUs.

- Localization time in the workshop (LRU only): Accounts for the localization time in the workshop and only applies again to RepAIR-1, 2 and it is set to 0.5 TUs.

- Mean time to detect (MTTD) in the workshop (LRU only): Considers the average time to detect the failure mode when the LRU has been dispatched to the workshop. The parameter is set to 3 TUs for RepAIR-1, 2.

\subsection{Case Cost and Downtime}

The previous sections described all the parameters that have to be taken into account (input probabilities, input costs and input times), and Figure 1 defines the probability of each possible case.

In order to calculate the total cost and downtime for all the scenarios, the cost and downtime of each possible case has to be calculated. There are a total of 28 cases. Thus, to avoid excessively large tables in the paper, please refer to Appendix A to check the associated cost $C_{i}$ and downtime $D_{i}$ of each case.

The average cost and downtime of each case is computed by multiplying each $\operatorname{cost} C_{i}$ and downtime $D_{i}$ for each specific probability $P_{i}$, see Eq. (2). Additionally, the total $\operatorname{cost} C_{T}$ and downtime $D_{T}$ are computed by summing all the weighted cases costs $C_{i}{ }^{w}$ and downtimes $D_{i}{ }^{w}$.

$$
\left\{\begin{array}{l}
C_{i}^{w}=C_{i} \cdot P_{i} \\
D_{i}^{w}=D_{i} \cdot P_{i}
\end{array} \forall i \in(1-28)\right.
$$

\section{MAINTENANCE OPTIMISATION CASE STUDY}

The four maintenance scenarios defined in Sub-section 2.2 are modelled using the cost and downtime model described in Appendix A. This will allow us to discuss the qualitative differences between the four maintenance scenarios in terms 
of cost and downtime. The parameters used are described in Sub-section 2.3, 2.4 and 2.5.

These parameters consist of synthetic data based on assumptions and similar studies (Esperon-Miguez, 2013). Please refer to similar studies for a better understanding of the cost downtime model and how the parameters are selected. Therefore, the aim of the paper is to propose the optimization approach and to discuss the impact of IVHM on the four maintenance scenarios discussed in Sub-section 2.2. This includes the qualitative differences between components that are part of a LRU and those which are not. Additionally, how additive manufacturing can potentially reduce costs and repair times can be analyzed for single parts and LRUs.

The values of all the parameters for the case studies have been defined along with their description in Section 2 .

\subsection{Integration of Real-Time Monitoring with Optimization}

Section 2 provided a comprehensive description of the model that calculates the cost and downtime of the different repair procedures and IVHM systems. This section presents implementation of the cost and downtime model with a realtime monitoring for optimizing maintenance activities, especially when AM is used, by advising on the optimal repair procedure.

This optimization is done by integrating the cost and downtime model within a real-time environment to choose the most appropriate repair procedure based on the actual status of the airline or MRO organization and the aircraft when a failure alarm is triggered.

The advantage of using an optimized algorithm of this kind is that the assessment of the cost and downtime can be more precise than if average costs and times are used from historical data. Additionally, the cheapest and faster repair/replacement procedure can be selected depending on the availability of labour resources, materials, spare parts, etc. which are updated by the CAMO (airline or MRO organization).

This is particularly useful if there is limited capability for the standard repair procedure and it is overloaded, which would lead to significantly higher costs and repair times. The real-time application would take the updated costs and times into account and a more optimal repair procedure can be selected.

It should be noted that for this algorithm to be effective, it is assumed that all the parameters required as inputs in Section 2 are updated in real time. This is a particular challenging aspect as airline and MRO structures are complex and it may be challenging to calculate all these parameters for each workshop.
The real-time monitoring, optimization, maintenance cost and downtime models are developed in Matlab. The flowchart for real-time monitoring and optimization is described in the next section. The real-time application, developed in Matlab, also includes a Graphical User Interface (GUI) for the maintainer, where he can run the updated model and choose the optimal repair procedure. This type of optimization could be used by an airline or MRO organization to decide on the appropriate maintenance scenario, including using additive manufacturing.

\subsection{Real-Time Monitoring and Optimization Process}

The aim of real-time monitoring and optimization is to decide the optimal maintenance process when a problem arises in a component of any aircraft. When this problem occurs, this algorithm is triggered and the real-time application is executed.

The real-time monitoring system in an aircraft continuously provides information regarding the health of the system to the central node, which is the connection between the aircraft and the CAMO. The CAMO decides if further action is required based on the health condition monitoring system, failure information and threshold values, and if so, a preliminary Work Order (WO) is triggered and the optimal maintenance is computed, as shown in Figure 2.

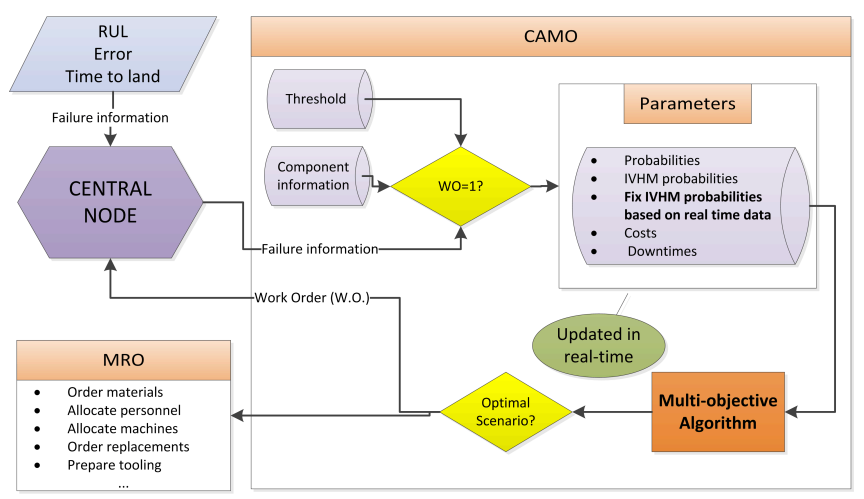

Figure 2. Real-time optimization flowchart

The maintenance optimization process shown in Figure 2 consists of the following:

- $\quad$ Trigger preliminary WO

- Update IVHM and failure probabilities

- Update resources

- Maintenance cost and downtime model

- Optimization algorithm

- Maintainer decision.

\section{Trigger Work Order}

The Work order can be triggered by: 
- Ground maintenance

- IVHM system.

The WO is launched immediately and sent to the real-time algorithm to decide the appropriate maintenance action if a problem has been detected on the ground by the maintainers or the problem is triggered by the health-monitoring system.

The following information is obtained from the healthmonitoring system for a specific part and failure mode and sent along with the WO:

- RUL (hours)

- Standard deviation with a 95\% confidence interval $\left(\sigma_{\text {RUL }}\right)$

- Hours to land ( $\left.\mathrm{T}_{\mathrm{LAND}}\right)$

- $\quad$ Aircraft ID, Flight ID, Part ID, Serial number.

The following is provided from the ground:

- Ground failure (Boolean value)

- Diagnosed (Boolean value).

The first action done by the real-time application is comparing the RUL to the threshold $\mathrm{RUL}_{\mathrm{LIM}}$, defined as the minimum time required to plan maintenance without compromising the availability of the aircraft. It should be noted that the standard deviation of the RUL $\left(\sigma_{\text {RUL }}\right)$ should be taken into account to ensure a certain degree of confidence in the RUL prediction, in this case a $95 \%$ confidence interval. However, confidence in the RUL prediction keeps increasing as RUL approaches 0 .

The alarm can be classified as ground failure, long-term predicted failure, short-term predicted failure, diagnosed failure and undiagnosed failure. See Eqs. (3-6)

$$
\begin{aligned}
& \text { if }\left\{\begin{array}{c}
\left(R U L-\sigma_{R U L}\right)>T_{L A N D} \\
\text { and } \\
\left(R U L-\sigma_{R U L}\right)>R U L_{L I M}
\end{array}\right\} \Rightarrow \text { long - term prog } \\
& \text { if }\left\{\begin{array}{c}
\left(R U L-\sigma_{R U L}\right)<T_{L A N D} \\
o r \\
\left(R U L-\sigma_{R U L}\right)<R U L_{L I M}
\end{array}\right\} \Rightarrow \text { short - term prog } \\
& \text { if }\left\{\begin{array}{c}
R U L=0 \\
\text { or } \\
\text { ground and diagnosed }
\end{array}\right\} \Rightarrow \text { diagnosed failure } \\
& \text { if }\left\{\begin{array}{c}
\text { ground failure } \\
\text { and } \\
\text { not diagnosed }
\end{array}\right\} \Rightarrow \text { undiagnosed failure }
\end{aligned}
$$

\section{Update IVHM and Failure Probabilities}

The CAMO should decide what the optimal maintenance procedure is by computing the model described in Section 2. However, in the real-time application, the cause of the failure is known and a more accurate estimation can be obtained by modifying the IVHM and failure probabilities as follows:

- $\quad$ Probability of failure $\left(\mathrm{P}_{-} \mathrm{S}\right)$ :

In a real-time scenario, the WO is triggered because a failure has been detected. Thus, the maintenance action will be executed and the probability of not having a failure and not having a false alarm is 0 . Therefore, $\mathrm{P}$ S should be updated accordingly, as shown in Eq. ( $\overline{7})$, where $P_{S}{ }^{*}$ is the average probability of a failure. It should be noted that if $\mathrm{P}_{-} \mathrm{S}$ is assumed equal to 1 , the possibility of a false alarm would not be considered.

$$
\text { if }(W O=1) \Rightarrow P_{S}=1-\left(1-P_{S}^{*}\right) P_{F A}
$$

- IVHM Probabilities (P_LP, P_SP, P_FN):

The IVHM probabilities (with the exception of $P_{-}$FA) are modified based on the type of failure previously. For instance, if the failure is considered long-term predicted with a 95\% confidence interval, the application assumes that it will consist in planned maintenance without affecting the availability of the aircraft. This can be done by modifying the IVHM probabilities, as shown in Eqs. (8-11).

if long - term prog. $\Rightarrow P_{L P}=0$

if short-term prog. $\Rightarrow P_{L P}=1, P_{S P}=0$

if diagnosed $\Rightarrow P_{L P}=1, P_{S P}=1, P_{F N}=0$

if undiagnosed $\Rightarrow P_{L P}=1, P_{S P}=1, P_{F N}=1$

\section{Update Resources}

The resources of the MRO organization vary over time and ultimately affect its costs and repair times. For strategic planning, these parameters should be estimated. However, in the ideal scenario of real-time optimized maintenance, these costs and times will change over time and can be tracked to have a more accurate cost and downtime estimation.

In this application, it is assumed that the MRO organization can estimate the repair procedure costs and times based on the availability of its resources and update the parameters of the maintenance and cost model accordingly.

The application and model retrieve the parameters from a spreadsheet to facilitate the modification of the database without interfering with the algorithm by the MRO organization. These values can easily be modified via web service or by any other means. 


\section{Maintenance Cost and Downtime Model}

Once all the parameters have been updated, the maintenance cost and downtime model is executed and the total cost and downtime of each repair procedure are obtained.

An extensive description of the maintenance cost and downtime model can be found in section 2. For the realapplication, the same model is implemented but only the actual IVHM scenario is considered.

\section{Health Monitoring and Optimization Algorithm}

The real-time health monitoring and optimization algorithm, shown in Figure 2, not only provides the estimated costs and downtimes. It also advises on the optimal maintenance process. The flowchart has been implemented in Matlab. The decision consists of a multi-objective algorithm where both cost and downtime should be minimized. However, the relative importance of each is not trivial.

The optimization would depend on the entity that acts as the CAMO. An airline may prioritize availability over cost, whereas an airline or MRO organization without an availability-based contract may prioritize low costs over high availability.

In this application, it is assumed that availability is prioritized over cost. Given the $\operatorname{cost} C_{k}$ and downtime $D_{k}$ of each repair option $k$, the algorithm considers that cost is relevant if downtime is reduced less than $50 \%$ and only if cost is reduced more than a $50 \%$ as well. Otherwise, the optimal option is based on the minimum downtime.

It should be noted that this simplified algorithm has been developed to take into account both cost and downtime as relevant factors choosing the optimal repair procedure and test the performance of the real-time application. The importance of these two variables ultimately depends on the user of the application.

\section{Maintainer Decision}

The results of the optimized model (cost and downtime) of each possible repair procedure along with the advised option obtained by the optimization algorithm are displayed in the GUI. In addition, the data obtained from the healthmonitoring system can be consulted as well in the GUI.

The person responsible to decide the optimal repair procedure can accept the recommended repair procedure or override the decision and select any other option based on the data provided by the model and his experience.

Once the maintainer selects the repair action, the WO is sent to the MRO organization, which will prepare the process, allocate materials, personnel, etc.

\subsection{Graphical User Interface}

This section describes the functionalities of the real-time application and how the user interacts with it. The GUI is shown in Figure 3.

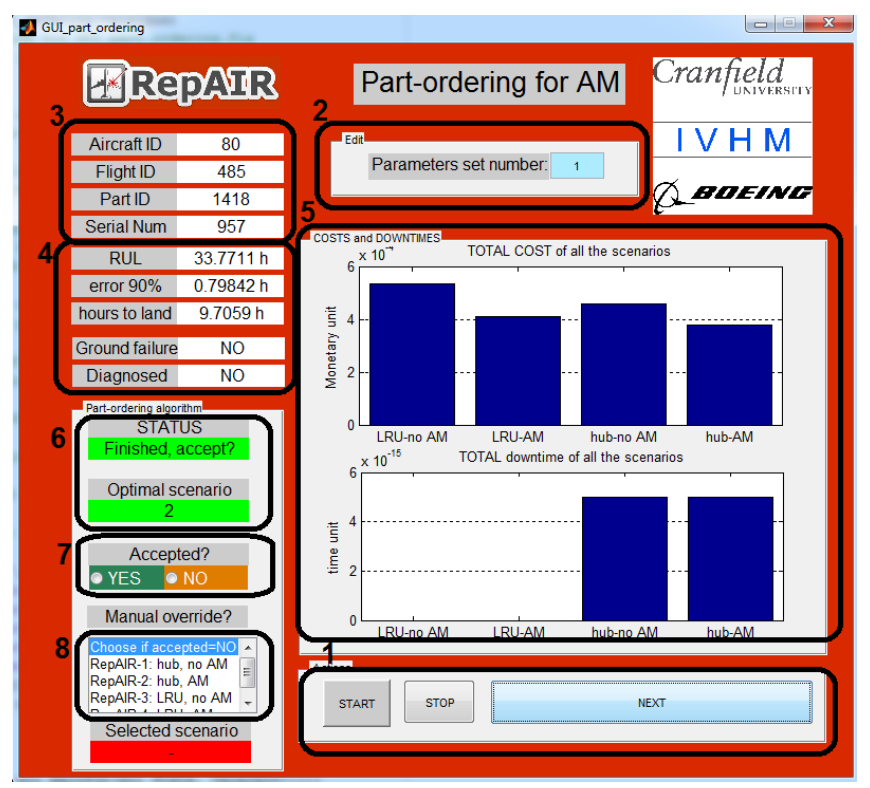

Figure 3. Maintainer graphical user interface

This applicaiton integrates all the functionalities described in Section Error! Reference source not found.. The maintainer interacts with the GUI by using the actions "START","STOP","NEXT" (see 1-Figure 3) to go though all the alarms that have to be analyzed.

The set of parameters for the maintainance cost and downtime model is defined in 2 in Figure 3. The aircraft, flight and part ID are provided along with the serial number for each failure, as shown in 3 in Figure 3.

The IVHM data and information from the ground are given in 4 in Figure 3. This information is used by the application to decide the type of alarm, update the parameters and execute the maintainence cost and downtime algorithm (see Sub-section Error! Reference source not found.).

The results, total cost $C_{k}$ and total downtime $D_{k}$, where $k$ is each repair alternative, are represented using bars as shown in 5 in Figure 3. The optimal scenario selected by the algorithm is also shown on the screen (see 6 in Figure 3) along with the status of the real-time application.

With all this information, the maintainer can manually go though all the issues, evaluate the expected cost and downtime with accurate information about the status of the aircraft and the maintenance capabilities, and decide what is the optimal repair procedure, either accepting the 
recommended optimal procedure (see 7 in Figure 3) or reject it and override it manually based on his expertise (see 8 in Figure 3).

Summarizing, the application provides a visualization tool to help the maintainer in the decision of the optimal repair procedure by giving additional relevant information (expected cost and downtime, health-monitoring data, etc.) that would lead to better supported decisions regarding the repair of the damaged part.

\section{RESULTS}

A variety of results can be obtained from the model. The most relevant ones are the total cost per flying hour of each maintenance scenario and the total downtime per flying hour as well (see Figure 4 and Figure 5).

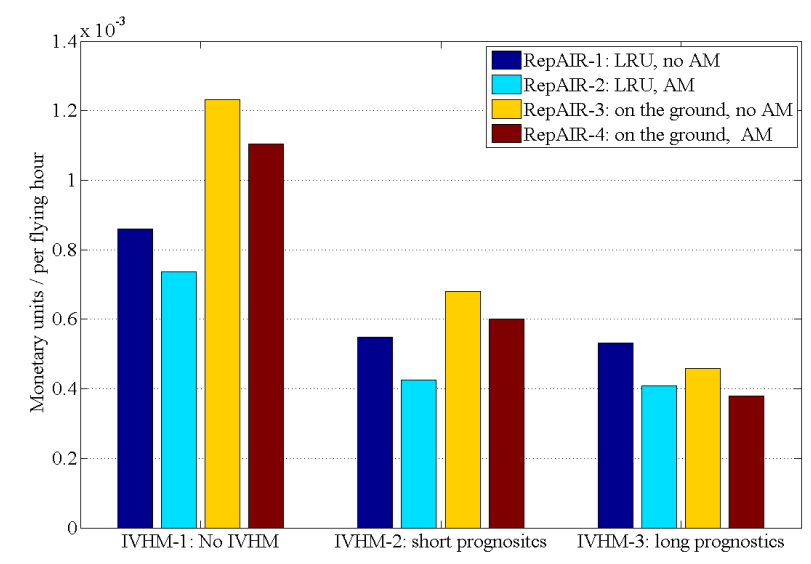

Figure 4. Total cost per flying hour of each scenario

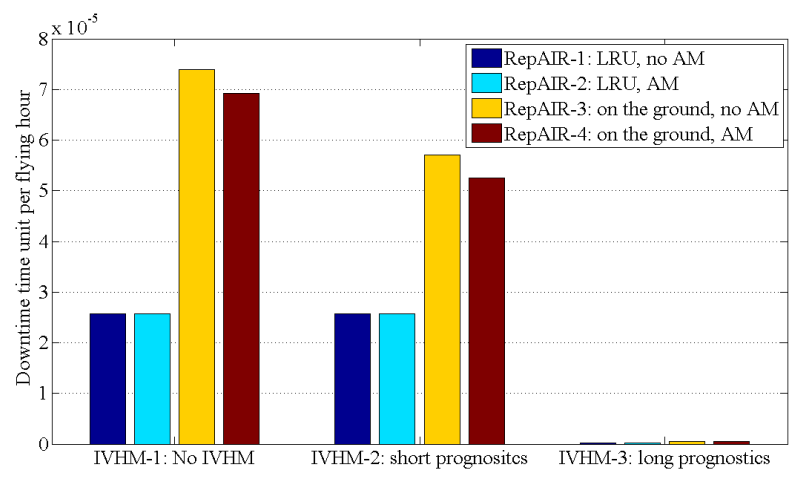

Figure 5. Total downtime per flying hour of each scenario

The cost and repair times for AM parts were assumed lower than traditional procedures. The impact on total cost is shown in Figure 4, where repairing the part by AM is cheaper than by traditional methods, regardless of doing it on the ground or in the workshop for LRUs. The reduction in time is not only reflected in the cost, but also in the downtime when the repair tasks are done on the ground (see Figure 5). However, downtime is identical for both repair procedures in LRU units because only assembly and disassembly of the LRU is done on the ground.

For the case studies presented in this paper, repairing the component as part of a LRU is more convenient than repairing the component on the ground (see Figure 4) and the downtime is significantly reduced (see Figure 5) because no repair procedure is done on the ground. However, to decide whether having a LRU is more profitable than repairing each single sub-component on the ground, the same study should be done over all the components and failure modes of the LRU and cannot be assessed by this single-part example.

Regarding the short-term IVHM system, it can be shown that having a short-term prognostics IVHM system would lead to a significant reduction of the total cost (see Figure 4) due to the avoidance of secondary damage, plus the diagnostics advantages of reduced MTTD.

Additionally, downtime is also reduced if the component is repaired on the ground (RepAIR-3, 4) as shown in Figure 5 because in that case, the downtime is directly affected by the reduced MTTR. However, downtime remains unaffected for LRUs because the repair procedure does affect the availability of the aircraft.

Finally, the advantages of using a long-term prognostics IVHM system are both cost and downtime reduction. A reliable IVHM system would minimize the undetected failures early in advance to plan the maintenance and therefore, the availability of the aircraft would not be compromised, as shown in Figure 5. The costs are also reduced when using a long-term IVHM system because planned maintenance is not as expensive but the cost reduction is not as significant as the downtime reduction when compared to a short-term prognostics IVHM system (see Figure 4).

\section{DISCUSSION AND CONCLUSIONS}

The results show that IVHM systems with low false alarm rates can significantly reduce the cost of the maintenance. However, the extra effort required to have reliable long-term prognostics is reflected in significant downtime reduction but not as much in terms of cost. Therefore, the benefits of developing IVHM capabilities are not identical for all the stakeholders, e.g. the airline would consider higher availability a priority and would be interested in long-term prognostics capabilities, whereas an MRO organization, without an availability-based contract, would not get any benefit of the higher availability apart from the satisfaction of its client.

From an IVHM perspective, for short-term prognostics (IVHM-2) and no IVHM capabilities (IVHM-1), repairing the component as part of a LRU is more efficient than as a single unit on the ground. However, the cost of the LRU option is higher for the long-term prognostics (IVHM-3) 
than repairing it on the ground. The reasons are the reduced costs and MTTR for planned maintenance compared to unplanned maintenance on the ground when a failure is detected early in advance.

From a repair technology perspective, the improvement of the technology, for instance, by using AM, would help to reduce the cost and time of the repair procedure. Additionally, delivery times and the probability of not having stock can be reduced because at least in the case of $\mathrm{AM}$, the powder is the only resource that has to be in stock. This is reflected in the reduced total costs for all the scenarios. However, downtime is only reduced if the component is repaired on the ground, thereby affecting the availability of the aircraft. This aspect should be taken into account when considering investing in new technologies for LRUs because a better repair procedure does not increase the availability of the aircraft.

From the previous discussion, the following conclusions can be extracted:

- Robust IVHM systems can significantly reduce maintenance cost (even short-term prognostics).

- Robust long-term prognostics IVHM systems significantly reduce downtime but that is not directly beneficial for all stakeholders.

- The use of IVHM affects maintenance costs and can have an influence on the optimal repair procedure.

- New technologies that reduce repair costs and the MTTR lead to lower total costs but will not reduce downtime unless the part is repaired on the ground.

The findings presented above are relevant but it should be noted that the main contributions of this paper are the comprehensive description of the maintenance cost and downtime model and the real-time application to select the optimal maintenance procedure, including additive manufacturing, based on up-to-date resources and the actual condition of the aircraft.

The maintenance cost and downtime model takes into consideration the use of new technologies and capabilities, e.g. it considers the probability of having stock and average delivery times. Moreover, it accounts for the unique differences between repairing a component as part of a LRU in the workshop and repairing it on the ground and uses the health-monitoring system installed in the aircraft.

The real-time optimization algorithm presents a novel approach to conduct maintenance and aims to exploit all the advantages of health-monitoring systems and different repair technologies and procedures. It provides an integrated platform and a GUI for the maintainer to choose the optimal maintenance option based on a model that considers the actual health status of the aircraft and the up-to-date available resources of the MRO organization.
This real-time application supports the maintainer in repair decision-making and, in combination with his expertise, can lead to lower repair costs and higher availabilities.

\section{ACKNOWLEDGEMENT}

The research leading to these results has received funding from the European Union Seventh Framework Programme (FP7/2007-2013) under Grant Agreement n605779 (project RepAIR). The text reflects the authors' views. The European Commission is not liable for any use that may be made of the information contained therein. For further information, see http://www.rep-air.eu/.

\section{REFERENCES}

Datta, K.. \& Squires, D. (2004). A methodology to quantify some IVHM requirements during RLV conceptual design. Reliability and Maintainability, 2004 Symposium-RAMS, January 2004, pp. 485-491.

Dunsdon, J. \& Harrington, M. (2008). The application of Open system architecture for condition based maintenance to complete IVHM. IEEE Aerospace Conference. March 2008, Big Sky, MT., pp. 1-9.

EASA (2003), Commission Regulation (EC) No 2042/2003 of 20 November 2003 on continuing airworthiness of aircraft and aeronautical products, parts and appliances, and on the approval of organisations and personnel involved in these tasks. Official Journal of the European Union, November 2003, pp. L 315/1 to L315/165.

Esperon-Miguez, M. (2013). Financial and risk assessment and selection of health monitoring system design options for legacy aircraft. Doctoral Dissertation. Cranfield University. United Kingdom.

Jennions, I. K. (2011). Integrated vehicle health management perspectives on an emerging filed. SAE International, Warrendale, Pa, ISBN: 0768064325.

Kumar, P. \& Varkey, C. C. (2012). Designing line replaceable units from the maintainability perspective. White paper of Guest-global. https://www.questglobal.com/wp-content/uploads/2015/07/DesigningLine-Replaceable-Units-from-the-MaintainabilityPerspective.pdf

Uriondo, A., Esperon-Miguez, M. \& Perinpanayagam, S. (2014), The present and future of additive Manufacturing in the aerospace sector: A review of important aspects. Proceedings of the Institution of Mechanical Engineers, Part G: Journal of Aerospace Engineering, vol. 229, pp. 2132-2147.

Xia, L., Rong, L., Zhao, M., Wang, L. \& Man, Q. (2010). Research on Open System Architecture for equipment health management based on OSA-CBM. IEEE International Conference on Intelligent Computing and Intelligent Systems, ICIS, October 2010, Xiamen, Vol. 2, pp.246-250. 


\section{APPENDIX A}

Table 6. Costs of each case

\begin{tabular}{|c|c|}
\hline Case & Equation \\
\hline 1 & $\begin{array}{l}C_{1}=C_{S C}+C_{S C L} \cdot M T T R+C_{R U L L}+C_{F T}+ \\
C_{L O-L R U}+C_{R-L R U}+C_{L A-L R U} \cdot\left(M T T R_{L R U}+\right. \\
\left.M T T D_{L R U}+T_{L-L R U}\right)\end{array}$ \\
\hline 2 & $\begin{array}{l}C_{2}=C_{U C}+C_{U C L} \cdot M T T R+C_{R U L S}+C_{F T}+C_{L I}+ \\
C_{C}+C_{L O-L R U}+C_{R-L R U}+C_{L A-L R U} \cdot\left(M T T R_{L R U}+\right. \\
\left.M T T D_{L R U}+T_{L-L R U}\right)\end{array}$ \\
\hline $\begin{array}{l}2-\mathrm{B} \\
(23)\end{array}$ & $\begin{array}{l}C_{23}=C_{U C}+C_{U C L} \cdot M T T R+C_{R U L S}+C_{F T}+C_{L I}+ \\
C_{C}+C_{L O-L R U}+C_{R-L R U}+C_{L A-L R U} \cdot\left(M T T R_{L R U}+\right. \\
\left.M T T D_{L R U}+T_{L-L R U}\right)\end{array}$ \\
\hline 3 & $\begin{array}{l}C_{3}=C_{U C}+C_{U C L} \cdot M T T R+C_{R U L S}+C_{F T}+ \\
C_{L O-L R U}+C_{R-L R U}+C_{L A-L R U} \cdot\left(M T T R_{L R U}+\right. \\
\left.M T T D_{L R U}+T_{L-L R U}\right)\end{array}$ \\
\hline 4 & $C_{4}=($ undefined $)$ \\
\hline 5 & $\begin{array}{l}C_{5}=C_{U C}+C_{U C L} \cdot M T T R+C_{S D}+C_{L I}+C_{C}+C_{F T}+ \\
C_{L O-L R U}+C_{R-L R U}+C_{L A-L R U} \cdot\left(M T T R_{L R U}+\right. \\
\left.M T T D_{L R U}+T_{L-L R U}\right)\end{array}$ \\
\hline $\begin{array}{l}5-B \\
(24)\end{array}$ & $\begin{array}{l}C_{24}=C_{U C}+C_{U C L} \cdot M T T R+C_{S D}+C_{L I}+C_{C}+ \\
C_{F T}+C_{L O-L R U}+C_{R-L R U}+C_{L A-L R U} \cdot\left(M T T R_{L R U}+\right. \\
\left.M T T D_{L R U}+T_{L-L R U}\right)\end{array}$ \\
\hline 6 & $\begin{array}{l}C_{6}=C_{U C}+C_{U C L} \cdot M T T R+C_{S D}+C_{F T}+C_{L O-L R U}+ \\
C_{R-L R U}+C_{L A-L R U} \cdot\left(M T T R_{L R U}+M T T D_{L R U}+\right. \\
\left.T_{L-L R U}\right)\end{array}$ \\
\hline 7 & $\begin{array}{l}C_{7}=C_{U C}+C_{U C L} \cdot M T T R+C_{S D}+C_{L I}+C_{C}+C_{F T}+ \\
C_{L O-L R U}+C_{R-L R U}+C_{L A-L R U} \cdot\left(M T T R_{L R U}+\right. \\
\left.M T T D_{L R U}+T_{L-L R U}\right)\end{array}$ \\
\hline $\begin{array}{l}7-B \\
(25)\end{array}$ & $\begin{array}{l}C_{25}=C_{U C}+C_{U C L} \cdot M T T R+C_{S D}+C_{L I}+C_{C}+ \\
C_{F T}+C_{L O-L R U}+C_{R-L R U}+C_{L A-L R U} \cdot\left(M T T R_{L R U}+\right. \\
\left.M T T D_{L R U}+T_{L-L R U}\right)\end{array}$ \\
\hline 8 & $\begin{array}{l}C_{8}=C_{U C}+C_{U C L} \cdot M T T R+C_{S D}+C_{F T}+C_{L O-L R U}+ \\
C_{R-L R U}+C_{L A-L R U} \cdot\left(M T T R_{L R U}+M T T D_{L R U}+\right. \\
\left.T_{L-L R U}\right)\end{array}$ \\
\hline 9 & $\begin{array}{l}C_{9}=C_{U C}+C_{U C L} \cdot M T T R+C_{S D}+C_{F T}+C_{L O-L R U}+ \\
C_{R-L R U}+C_{L A-L R U} \cdot\left(M T T R_{L R U}+M T T D_{L R U}+\right. \\
\left.T_{L-L R U}\right)\end{array}$ \\
\hline 10 & $C_{10}=N A($ undefined $)$ \\
\hline 11 & $\begin{array}{l}C_{11}=C_{U C}+C_{U C L} \cdot\left(M T T R+M T T D+T_{L}\right)+C_{S D}+ \\
C_{L I}+C_{C}+C_{F T}+C_{L O-L R U}+C_{R-L R U}+C_{L A-L R U} \\
\left(M T T R_{L R U}+M T T D_{L R U}+T_{L-L R U}\right)\end{array}$ \\
\hline $\begin{array}{l}11-B \\
(26)\end{array}$ & $\begin{array}{l}C_{26}=C_{U C}+C_{U C L} \cdot\left(M T T R+M T T D+T_{L}\right)+C_{S D}+ \\
C_{L I}+C_{C}+C_{F T}+C_{L O-L R U}+C_{R-L R U}+C_{L A-L R U} \\
\left(M T T R_{L R U}+M T T D_{L R U}+T_{L-L R U}\right)\end{array}$ \\
\hline 12 & $\begin{array}{l}C_{12}=C_{U C}+C_{U C L} \cdot\left(M T T R+M T T D+T_{L}\right)+ \\
C_{R U L S}+C_{S D}+C_{F T}+C_{L O-L R U}+C_{R-L R U}+C_{L A-L R U} \\
\left(M T T R_{L R U}+M T T D_{L R U}+T_{L-L R U}\right)\end{array}$ \\
\hline 13 & $\begin{array}{l}C_{13}=C_{U C}+C_{U C L} \cdot\left(M T T R+M T T D+T_{L}\right)+C_{S D}+ \\
C_{L I}+C_{C}+C_{F T}+C_{L O-L R U}+C_{R-L R U}+C_{L A-L R U} \\
\left(M T T R_{L R U}+M T T D_{L R U}+T_{L-L R U}\right)\end{array}$ \\
\hline $\begin{array}{l}13-\mathrm{B} \\
(27)\end{array}$ & $\begin{array}{l}C_{27}=C_{U C}+C_{U C L} \cdot\left(M T T R+M T T D+T_{L}\right)+C_{S D}+ \\
C_{L I}+C_{C}+C_{F T}+C_{L O-L R U}+C_{R-L R U}+C_{L A-L R U} \\
\left(M T T R_{L R U}+M T T D_{L R U}+T_{L-L R U}\right)\end{array}$ \\
\hline 14 & $\begin{array}{l}C_{14}=C_{U C}+C_{U C L} \cdot\left(M T T R+M T T D+T_{L}\right)+C_{S D}- \\
+C_{F T}+C_{L O-L R U}+C_{R-L R U}+C_{L A-L R U} \cdot\left(M T T R_{L R U}+\right. \\
\left.M T T D_{L R U}+T_{L-L R U}\right)\end{array}$ \\
\hline 15 & $C_{15}=C_{U C L} \cdot\left(M T T D+T_{L}\right)$ \\
\hline
\end{tabular}

\begin{tabular}{ll}
\hline $15-\mathrm{B}$ & $C_{28}=C_{U C L} \cdot\left(M T T D+T_{L}\right)$ \\
$(28)$ & \\
16 & $C_{16}=C_{U C L} \cdot\left(M T T D+T_{L}\right)$ \\
17 & $C_{17}=0$ \\
18 & $C_{18}=C_{S C}+C_{S C L} \cdot M T T D+C_{L I}+C_{C}+C_{L O-L R U}+$ \\
& $C_{R-L R U}+C_{L A-L R U} \cdot\left(M T T D_{L R U}+T_{L-L R U}\right)$ \\
19 & $C_{19}=C_{S C}+C_{S C L} \cdot M T T D+C_{L O-L R U}+$ \\
& $C_{R-L R U}+C_{L A-L R U} \cdot\left(M T T D_{L R U}+T_{L-L R U}\right)$ \\
20 & $C_{20}=C_{S C}+C_{S C L} \cdot M T T D+C_{L I}+C_{C}+C_{L O-L R U}+$ \\
& $C_{R-L R U}+C_{L A-L R U} \cdot\left(M T T D_{L R U}+T_{L-L R U}\right)$ \\
21 & $C_{21}=C_{S C}+C_{S C L} \cdot M T T D+C_{L O-L R U}+$ \\
& $C_{R-L R U}+C_{L A-L R U} \cdot\left(M T T D_{L R U}+T_{L-L R U}\right)$ \\
22 & $C_{22}=C_{S C}+C_{S C L} \cdot M T T D+C_{L O-L R U}+$ \\
& $C_{R-L R U}+C_{L A-L R U} \cdot\left(M T T D_{L R U}+T_{L-L R U}\right)$ \\
\hline &
\end{tabular}


Table 7. Downtimes of each case

\begin{tabular}{|c|c|}
\hline Case & Equation \\
\hline 1 & $D_{1}=0$ \\
\hline 2 & $\begin{array}{l}D_{2} \\
=M T T R+T_{\text {Check }}+T_{\text {technical-delay }}+T_{\text {admin-delay }} \\
+T_{\text {logistic-delay }}\end{array}$ \\
\hline $\begin{array}{l}2-\mathrm{B} \\
(23)\end{array}$ & $\begin{array}{l}D_{23} \\
=M T T R+T_{\text {Check }}+T_{\text {technical-delay }}+T_{\text {admin-delay }} \\
+T_{\text {logistic-delay }}+T_{\text {no-stock }}\end{array}$ \\
\hline 3 & $D_{3}=0$ \\
\hline 4 & $D_{4}=N A($ undefined $)$ \\
\hline 5 & $\begin{array}{l}D_{5} \\
=M T T R+T_{\text {Check }}+T_{\text {technical-delay }}+T_{\text {admin-delay }} \\
+T_{\text {logistic-delay }}\end{array}$ \\
\hline $\begin{array}{l}5-B \\
(24)\end{array}$ & $\begin{array}{l}D_{24} \\
=M T T R+T_{\text {Check }}+T_{\text {technical-delay }}+T_{\text {admin-delay }} \\
+T_{\text {logistic-delay }}+T_{\text {no-stock }}\end{array}$ \\
\hline 6 & $D_{6}=0$ \\
\hline 7 & $\begin{array}{l}D_{7} \\
=M T T R+T_{\text {Check }}+T_{\text {technical-delay }}+T_{\text {admin-delay }} \\
+T_{\text {logistic-delay }}\end{array}$ \\
\hline $\begin{array}{l}7-B \\
(25)\end{array}$ & $\begin{array}{l}D_{25} \\
=M T T R+T_{\text {Check }}+T_{\text {technical-delay }}+T_{\text {admin-delay }} \\
+T_{\text {logistic-delay }}+T_{\text {no-stock }}\end{array}$ \\
\hline 8 & $D_{8}=0$ \\
\hline 9 & $D_{9}=0$ \\
\hline 10 & $D_{10}=0$ \\
\hline 11 & $\begin{array}{l}D_{11} \\
=M T T R+M T T D+T_{L}+T_{\text {Check }}+T_{\text {technical-delay }} \\
+T_{\text {admin-delay }}+T_{\text {logistic-delay }}\end{array}$ \\
\hline $\begin{array}{l}11-B \\
(26)\end{array}$ & $\begin{array}{l}D_{26} \\
=M T T R+M T T D+T_{L}+T_{\text {Check }}+T_{\text {technical-delay }} \\
+T_{\text {admin-delay }}+T_{\text {logistic-delay }}+T_{\text {no-stock }}\end{array}$ \\
\hline 12 & $D_{12}=0$ \\
\hline 13 & $\begin{array}{l}D_{13} \\
=M T T R+M T T D+T_{L}+T_{\text {Check }}+T_{\text {technical-delay }} \\
+T_{\text {admin-delay }}+T_{\text {logistic-delay }}\end{array}$ \\
\hline $\begin{array}{l}13-\mathrm{B} \\
(27)\end{array}$ & $\begin{array}{l}D_{27} \\
=M T T R+M T T D+T_{L}+T_{\text {Check }}+T_{\text {technical-delay }} \\
+T_{\text {admin-delay }}+T_{\text {logistic-delay }}+T_{\text {no-stock }}\end{array}$ \\
\hline 14 & $D_{14}=0$ \\
\hline 15 & $\begin{array}{l}D_{15} \\
=M T T D+T_{L}+T_{\text {Check }}+T_{\text {technical-delay }} \\
+T_{\text {admin-delay }}+T_{\text {logistic-delay }}\end{array}$ \\
\hline $\begin{array}{l}15-\mathrm{B} \\
(28)\end{array}$ & $\begin{array}{l}D_{28} \\
=M T T D+T_{L}+T_{\text {Check }}+T_{\text {technical-delay }} \\
+T_{\text {admin-delay }}+T_{\text {logistic-delay }}+T_{\text {no-stock }}\end{array}$ \\
\hline 16 & $D_{16}=0$ \\
\hline 17 & $D_{17}=0$ \\
\hline 18 & $D_{18}=M T T D$ \\
\hline 19 & $D_{19}=M T T D$ \\
\hline 20 & $D_{20}=M T T D$ \\
\hline
\end{tabular}

\begin{tabular}{ll}
\hline 21 & $D_{21}=$ MTTD \\
22 & $D_{22}=0$ \\
\hline
\end{tabular}

